\title{
THE EFFECT OF THE MAGNETIC FIELD ON THE RAYLEIGH-TAYLOR INSTABILITY IN A COUPLE-STRESS FLUID
}

\author{
K.B. CHAVARADDI \\ Department of Mathematics, S.S. Government First Grade College \\ Nargund, INDIA \\ E-mail: ckrishna2002@yahoo.com \\ V.B. AWATI \\ Department of Mathematics, Rani Channamma University \\ Belgaum, INDIA \\ M.M. NANDEPPANAVAR ${ }^{*}$ \\ Department of Mathematics, Government College \\ Gulbarga - 585105, Karnataka, INDIA \\ E-mail: nandeppanavarmm@gmail.com \\ P.M. GOUDER \\ K.L.E's Dr. M. S. Sheshagiri College of Engineering and Technology \\ Belgaum, INDIA \\ E-mail: nandeppanavarmm@gmail.com
}

\begin{abstract}
In this study we examine the effect of the magnetic field parameter on the growth rate of the Rayleigh-Taylor instability (RTI) in a couple stress fluids. A simple theory based on fully developed flow approximations is used to derive the dispersion relation for the growth rate of the RTI. The general dispersion relation obtained using perturbation equations with appropriate boundary conditions will be reduced for the special cases of propagation and the condition of instability and stability will be obtained. In solving the problem of the R-T instability the appropriate boundary conditions will be applied. The couple-stress parameter is found to be stabilizing and the influence of the various parameters involved in the problem on the interface stability is thoroughly analyzed. The new results will be obtained by plotting the curves between the dimensionless growth rate and the dimensionless wave number for various physical parameters involved in the problem (viz. the magnetic field, couple-stress, porosity, etc.) in the problem. It is found that the magnetic field and couple-stress have a stabilization effect whereas the buoyancy force (surface tension) has a destabilization effect on the RT instability in the presence of porous media.
\end{abstract}

Key words: RTI, couple-stress fluid, magnetic field, porous media, growth rate.

\section{Introduction}

Interfacial instabilities in fluid systems are characterized by material interpenetration and mixing at molecular scales due to perturbations occurring at material interfaces. Rayleigh-Taylor, Richtmyer-Meshkov, and Kelvin-Helmholtz instabilities are important hydrodynamic instabilities. The Rayleigh-Taylor instability is buoyancy-driven and occurs in fluid flows that contain species of differing molecular masses in the

\footnotetext{
* To whom correspondence should be addressed
} 
presence of acceleration, such as gravity or accelerating fronts. The Richtmyer-Meshkov instability is shockinduced and occurs due to the interaction between the shock wave and the perturbed material interface separating two fluids. The Kelvin-Helmholtz instability is shear-induced and occurs in a continuous fluid system where velocity shear is present or between two fluids due to a sufficient velocity difference across the interface. A detailed understanding of the processes and the consequences of interfacial instabilities have vast applications to real systems including the design of efficient, high-gain capsules for inertial confinement fusion (ICF) and the modelling of supernova implosions and explosions[1]. Such an understanding is becoming available due to current computational efforts.

A qualitative understanding of all three instabilities is an important first step in understanding the processes and effects involved with interfacial instabilities. A mathematical approach by applying the Navier-Stokes equations supports the qualitative analysis. One can then use these analyses to view the evolution of the instabilities. Rayleigh-Taylor instability(RTI): A simple explanation for the occurrence of the Rayleigh-Taylor instability deals with a normal room existing on earth with normal gravitational effects [2]. Suppose this room contains a volume of air above the floor, which is supporting a uniform layer of water that lies beneath the ceiling. The Rayleigh-Taylor instability (RTI) occurs when a heavy fluid is supported by a lighter one, when a heavy fluid is accelerated by a lighter one. Similar to pouring of water into an oil, the heavier fluid, once perturbed, streams to the bottom, pushing the light fluid aside. Richtmyer-Meshkov instability (RMI): A similar argument can be made for the Richtmyer-Meshkov instability. Suppose a perturbation exists at an interface between a heavy and a light fluid. Kelvin-Helmholtz instability(KHI): As with the two previous instabilities, the Kelvin-Helmholtz instability grows from an initial perturbation at the interface of two fluids of different densities.

The notion of RTI for a fluid in a gravitational field was first studied by Lord Rayleigh [3] and later applied to all accelerated fluids by Sir Geoffrey Taylor [4]. The RTI has been addressed in several studies owing to its importance in science, engineering and technology. RTI in hydrodynamics and magnetohydrodynamics has been extensively investigated (see Chandrashekar [5]). Bhatia [6] studied the stability of a plane interface separating two incompressible superposed conducting fluids of uniform density, when the whole system is under the influence of a uniform magnetic field. He carried out the stability analysis of two highly viscous fluids of equal kinematic viscosity and different uniform densities. The RTI of two viscoelastic (Oldroyd) superposed fluids was studied by Sharma and Sharma [7]. Also, the R-T instability between two fluids was investigated in the past by several authors due to the relevance of this problem in different media.

Nevertheless, much attention has not been given in the literature to the study of the RTI in a poorly conducting non-Newtonian fluid like a couple stress fluid with the effect of surface roughness. The couplestress effects are considered a result of the action of one part of a deforming body on its neighbourhood. Stokes [8] formulated the theory of a couple-stress fluid. The theory of Stokes [8] allows for the polar effects such as the presence of couple-stresses and body couples and has been applied to the study of some simple lubrication problems(see Sinha et al. [9], Bujurke and Jayaraman [10]). According to Stokes [8], couplestresses appear in fluids with very larger molecules. Since the long chain hylauronic acid molecules are found as additives in synovial fluids, Walicki and Walicka [11] modeled the synovial fluid as couple-stress fluid in human joints. The presence of small amounts of additives in a lubricant can improve the bearing performance by increasing the lubricant viscosity and thus producing an increase in the load capacity. This additive in a lubricant also reduces the coefficient of friction and increases the temperature range in which a bearing can operate. The RTI of two superposed infinitely conducting couple-stress fluids of uniform densities in a porous medium in the presence of a uniform magnetic field was a studied by Sunil et al. [13].

Recently, Chavaraddi et al. [14] studied the effect of boundary roughness on the RTI in a couplestress fluid in the absence of porous media. Much work has also been done on numerical simulations of both the RT and KH instabilities, taking into account the resulting magnetic field generation [15-18]. However, until now, all numerical investigations have been made for strongly oversimplified model flows, such as the classical 2D RT instability at an inert interface [15-17], which is far away from realistic ICF conditions.

Keeping in mind the importance of non-Newtonian (couple-stress) fluids in modern technology and industries as well as various applications mentioned above, in the present paper, we investigate the effect of 
the magnetic field on RTI in a poorly conducting couple-stress fluid layer bounded above by a porous layer in. The aim of this study is to see how the condition of instability and stability changes with changing the direction of the magnetic field. Also, we will discuss the effect of porosity, magnetic field, surface tension and viscosity on the R-T instability. Our aim is to study how the condition of stability as well as instability and growth rate changes with changes in these parameters.

The paper is organized in the following manner. In section 2 , the basic equations describing the dynamics of system which evolve in consequence of the combined effects magnetic field and couple-stress fluid of this hydrodynamical instability (RTI) are developed. The mathematical formulation subjected to the boundary and surface conditions is given in this section. In section 3, it is shown that the expression for the dispersion relation is derived using the basic equations with boundary and surface conditions. Analytical results regarding the saturation growth rates and and the corresponding maximum growth rate are presented in section 4. Finally, section 5 presents a brief conclusion of these results.

\section{Mathematical formulation}

The configuration can be seen in Fig.1. The system under consideration is composed of two incompressible couple-stress fluids and fluid saturated porous media separated by the plane $y=h$. Since gravity is present; the effective acceleration is in the positive $y$-direction (upwards). Therefore, the light fluid pushes on the heavy fluid (fluid saturated porous media). As long as the interface between fluids remains uniform, that is completely horizontal and perpendicular to the effective acceleration, the light fluid (couple stress fluid) has sufficient pressure to hold the heavy fluid up. However, small deviations are bound to occur at the interface. When the irregularities occur, portions of the interface lie higher than the average and therefore feel more pressure from the light fluid than is necessary to support the water. Therefore, the interface continues to rise in the spots. In the portions where the interface has dropped by small amounts below the average, more pressure is needed for support, and the interface drops further. Even the slightest perturbation will cause instability. If the situation were reversed, and the heavy fluid layer was on the floor supporting the volume of the light fluid, the pressure gradients reverse. In the portions of the interface that have risen, the heavy fluid will lower back to average, while the lowered portions will rise again. This qualitative analysis leads to the conclusive statement that the interface is stable if the heavy fluid pushes the light fluid and unstable if the light fluid pushes the heavy fluid. The unstable case is the Rayleigh-Taylor instability.

The upper fluid has density $\rho_{p}$ while the lower one has density $\rho_{f}$ and surface tension exists between the two fluids and is denoted by $\gamma$.

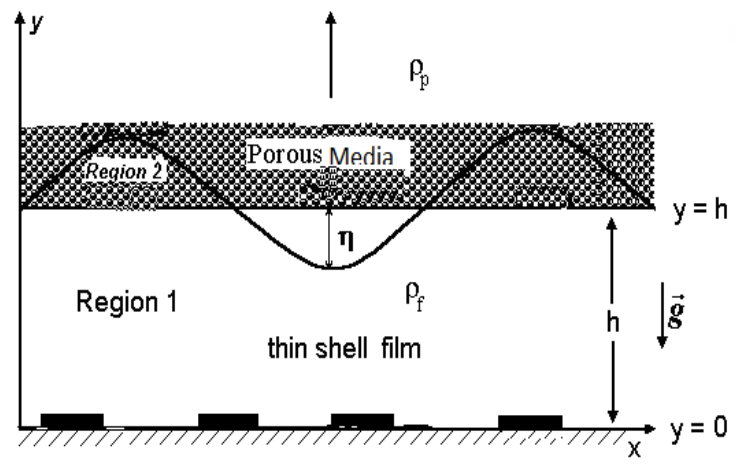

Fig.1. Physical configuration.

To investigate the problems posed in the paper the following combined lubrication and Stokes approximations are used: 
(i) The clear dense liquid is homogeneous and isotropic.

(ii) The film thickness $h$ is much smaller than the thickness $H$ of the porous layer bounded above the film. That is, $h \ll H$.

(iii) The Strouhal number $S$ is assumed to be negligibly small.

(iv) The surface elevation $\eta$ is assumed to be small compared to film thickness $h, \eta \ll h$.

(v) Non-uniform polarization and electric charge injection are negligible.

(vi) The fluid viscosity and thermal conductivity are assumed to be constant values.

Following these assumptions and approximations, the basic equations are

$$
\begin{aligned}
& \nabla \cdot \boldsymbol{q}=0 \\
& \rho_{f}\left[\frac{\partial \boldsymbol{q}}{\partial t}+(\boldsymbol{q} \cdot \nabla) \boldsymbol{q}\right]=-\nabla p+\mu_{f} \nabla^{2} \boldsymbol{q}+\mu_{\boldsymbol{o}}(\boldsymbol{J} \times \boldsymbol{H})-\lambda_{\boldsymbol{f}} \nabla^{4} \boldsymbol{q}
\end{aligned}
$$

\section{Maxwell's Equations}

$$
\nabla . \boldsymbol{E}=0, \quad \nabla \cdot \boldsymbol{H}=0, \quad \nabla \times \boldsymbol{E}=-\frac{\partial \boldsymbol{B}}{\partial t}, \quad \nabla \times \boldsymbol{H}=\boldsymbol{J}+\frac{\partial \boldsymbol{D}}{\partial t},
$$

and the auxiliary equations

$$
\boldsymbol{D}=\varepsilon_{0} \boldsymbol{E}, \quad \boldsymbol{B}=\mu_{0} \boldsymbol{H}, \quad \boldsymbol{J} \times \boldsymbol{B}=\sigma[\boldsymbol{E}+\boldsymbol{q} \times \boldsymbol{B}] \times \boldsymbol{B} .
$$

The physical quantities appearing in the above equations are defined in the nomenclature.

Boundary conditions:

i) no-slip condition: $\quad u=0$ at $y=0$.

ii) Saffman (1971) slip condition: $\frac{\partial u}{\partial y}=-\frac{\alpha_{p}}{\sqrt{k}} \quad$ at $\quad y=h$.

iii) kinematic condition:

$v=\frac{\partial \eta}{\partial t}+u \frac{\partial \eta}{\partial x} \quad$ at $\quad y=h$.

iv) dynamic condition:

$$
p=-\delta \eta-\gamma \frac{\partial^{2} \eta}{\partial x^{2}} \quad \text { at } \quad y=h
$$

v) couple-stress condition:

$$
\frac{\partial^{2} u}{\partial y^{2}}=0 \quad \text { at } \quad y=0 \& h
$$

Let us non-dimensionalize the above equations using

$$
x^{*}=\frac{x}{h}, \quad y^{*}=\frac{y}{h}, \quad u^{*}=\frac{u}{\delta h^{2} / \mu}, \quad v^{*}=\frac{v}{\delta h^{2} / \mu}, \quad p^{*}=\frac{p}{\delta h}, \quad \sigma_{p}^{*}=\frac{h}{\sqrt{k}}, \quad \eta^{*}=\frac{\eta}{h} .
$$

Following the assumptions and approximations as stated above (i.e., Stokes and lubrication approximations), assuming that the heavy fluid in the porous layer is almost static because of creeping flow approximation and substituting Eq.(2.10) into Eqs (2.1) and (2.2), we obtain (after neglecting the asterisks for simplicity)

$$
0=\frac{\partial u}{\partial x}+\frac{\partial v}{\partial y}
$$




$$
\begin{aligned}
& 0=-\frac{\partial p}{\partial x}+\frac{\partial^{2} u}{\partial y^{2}}-M_{0}^{2} \frac{\partial^{4} u}{\partial y^{4}}-M^{2} u \\
& 0=-\frac{\partial p}{\partial y}+\frac{\partial^{2} u}{\partial y^{2}}-M_{0}^{2} \frac{\partial^{4} u}{\partial y^{4}}-M^{2} u
\end{aligned}
$$

where $M_{0}$ - the couple-stress parameter and $M^{2}$ - the Hartmann number are defined in the nomenclature.

\section{Dispersion relation}

To find the dispersion relation, first we have to find the velocity distribution from Eq.(2.12) using the following boundary and surface conditions in addition to couple-stress boundary conditions.

(i) no-slip condition

$$
u=0 \quad \text { at } \quad y=0 .
$$

(ii) Saffmann-slip condition

$$
\frac{\partial u}{\partial y}=-\alpha_{p} \sigma_{p} u \quad \text { at } \quad y=1
$$

(iii) couple-stress conditions

$$
\frac{\partial^{2} u}{\partial y^{2}}=0 \quad \text { at } \quad y=0 \& 1
$$

(iv) kinematic condition

$$
v=\frac{\partial \eta}{\partial t} \quad \text { at } \quad y=1
$$

(v) dynamic condition

$$
p=-\eta-\frac{1}{B} \frac{\partial^{2} \eta}{\partial x^{2}} \quad \text { at } \quad y=1
$$

where B the Bond number, $\sigma_{p}$ - the porous parameter are defined in the nomenclature, and $\eta=\eta(x, y, t)$ is the elevation of the interface.

The solution of Eq.(2.12) subject to the above conditions is

$$
u=\left[C_{1} \cosh \left(\alpha_{1} y\right)+C_{2} \sinh \left(\alpha_{1} y\right)+C_{3} \cosh \left(\alpha_{2} y\right)+C_{4} \sinh \left(\alpha_{2} y\right)-\frac{1}{M^{2}}\right] P
$$

where

$$
P=\frac{\partial p}{\partial x}, \quad \alpha_{1}^{2}=\frac{1+\sqrt{1-4 M^{2} M_{0}^{2}}}{2 M_{0}^{2}}, \quad \alpha_{2}^{2}=\frac{1-\sqrt{1-4 M^{2} M_{0}^{2}}}{2 M_{0}^{2}}
$$




$$
\begin{aligned}
& C_{1}=\frac{P b_{1} \alpha_{2}^{2}}{\alpha_{1}^{2}-\alpha_{2}^{2}}, \quad C_{3}=\frac{P b_{1} \alpha_{1}^{2}}{\alpha_{1}^{2}-\alpha_{2}^{2}}, \\
& C_{2}=\frac{P\left(a_{3} a_{8} b_{1} \alpha_{1}^{2}-a_{4} a_{7} b_{1} \alpha_{1}^{2}-a_{8} b_{2} \alpha_{1}^{2}+a_{4} a_{5} b_{1} \alpha_{2}^{2}-a_{1} a_{8} b_{1} \alpha_{2}^{2}+a_{8} b_{2} \alpha_{2}^{2}\right)}{\left(a_{4} a_{6}-a_{2} a_{8}\right)\left(\alpha_{1}^{2}-\alpha_{2}^{2}\right)}, \\
& C_{4}=\frac{-P\left(a_{3} a_{6} b_{1} \alpha_{1}^{2}-a_{2} a_{7} b_{1} \alpha_{1}^{2}-a_{6} b_{2} \alpha_{1}^{2}+a_{2} a_{5} b_{1} \alpha_{2}^{2}-a_{1} a_{6} b_{1} \alpha_{2}^{2}+a_{6} b_{2} \alpha_{2}^{2}\right)}{\left(a_{4} a_{6}-a_{2} a_{8}\right)\left(\alpha_{1}^{2}-\alpha_{2}^{2}\right)} \\
& a_{1}=\alpha_{1} \sinh \left(\alpha_{1}\right)+\alpha_{p} \sigma_{p} \cosh \left(\alpha_{1}\right), \quad a_{2}=\alpha_{1} \cosh \left(\alpha_{1}\right)+\alpha_{p} \sigma_{p} \sinh \left(\alpha_{1}\right), \\
& a_{3}=\alpha_{2} \sinh \left(\alpha_{2}\right)+\alpha_{p} \sigma_{p} \cosh \left(\alpha_{2}\right), \quad a_{4}=\alpha_{2} \cosh \left(\alpha_{2}\right)+\alpha_{p} \sigma_{p} \sinh \left(\alpha_{2}\right), \\
& a_{5}=\alpha_{1}^{2} \cosh \left(\alpha_{1}\right), \quad a_{6}=\alpha_{1}^{2} \sinh \left(\alpha_{1}\right), a_{7}=\alpha_{7}^{2} \cosh \left(\alpha_{2}\right), a_{5}=\alpha_{2}^{2} \sinh \left(\alpha_{2}\right), \\
& b_{1}=\frac{1}{M^{2}}, \quad b_{2}=\frac{\alpha_{p} \sigma_{p}}{M^{2}} .
\end{aligned}
$$

After integrating Eq.(2.11) with respect to $y$ between $y=0$ and $l$ and using Eq.(3.6), we get

$$
v(I)=v_{l}=-\int_{0}^{l} \frac{\partial u}{\partial x} d y=\frac{\partial^{2} p}{\partial x^{2}} \Delta_{l}
$$

where

$$
\Delta_{1}=\frac{C_{1}}{\alpha_{1}} \sinh \left(\alpha_{1}\right)+\frac{C_{2}}{\alpha_{1}}\left(\cosh \left(\alpha_{1}\right)-1\right)+\frac{C_{3}}{\alpha_{2}} \sinh \left(\alpha_{2}\right)+\frac{C_{4}}{\alpha_{2}}\left(\cosh \left(\alpha_{2}\right)-1\right)-\frac{1}{M^{2}} .
$$

Then Eq.(3.4), using Eqs (3.5) and (3.7), becomes

$$
\frac{\partial \eta}{\partial t}=-\left[\frac{\partial^{2} \eta}{\partial x^{2}}+\frac{1}{B} \frac{\partial^{4} \eta}{\partial x^{4}}\right] \Delta_{l}
$$

To investigate the growth rate, $n$, of the periodic perturbation of the interface, we look for the solution of Eq.(3.8) in the form

$$
\eta=\eta(y) \exp \{i \ell x+n t\}
$$

where $\ell$ is the wave number and $\eta(y)$ is the amplitude of perturbation of the interface.

Substituting Eq.(3.9) into Eq.(3.8), we obtain the dispersion relation in the form 


$$
n=\ell^{2}\left(1-\frac{\ell^{2}}{B}\right) \Delta
$$

where

$$
\Delta=-\Delta_{l}
$$

The growth rate given by Eq.(3.10) is computed numerically for different values of parameters and the results are presented graphically in Figs 2-5.

\section{Results and discussion}

In this study we have shown the effect of physical parameters involved in the problem on the effect of the magnetic field on surface instability of RT type in a couple-stress fluid layers bounded above by a porous layer and below by a rigid boundary. Numerical calculations were performed to determine the growth rate at different wave numbers for various fluid properties such as the couple stress parameter $M_{0}$, Hartmann number $\mathrm{M}$, Bond number B and porous parameter $\sigma_{p}$. We have plotted the dimensionless growth rate of the perturbation against the dimensionless wave number for some of the cases only. In the linear stage, all perturbed values grow exponentially in agreement with the dispersion relation Eq.(3.10). At this stage the interface between the layers acquires a sinusoidal shape of small amplitude.

When all the input parameters are fixed we find that the higher the couple-stress parameter, the more stable the interface is. In Fig.2, we have plotted the growth rate against the wave number in the case where $\alpha_{p}=0.1, \sigma_{p}=4, B=0.02$ and $\mathrm{M}=5$ for different values of the couple-stress parameter $M_{0}$. Increasing the couple-stress ratio results in slightly increasing the critical wave number and decreasing the maximum growth rate this is because of the action of the body couples on the system. Thus, it has a stabilizing effect for the selected values of input parameters due to the increase in the couple-stress parameter.

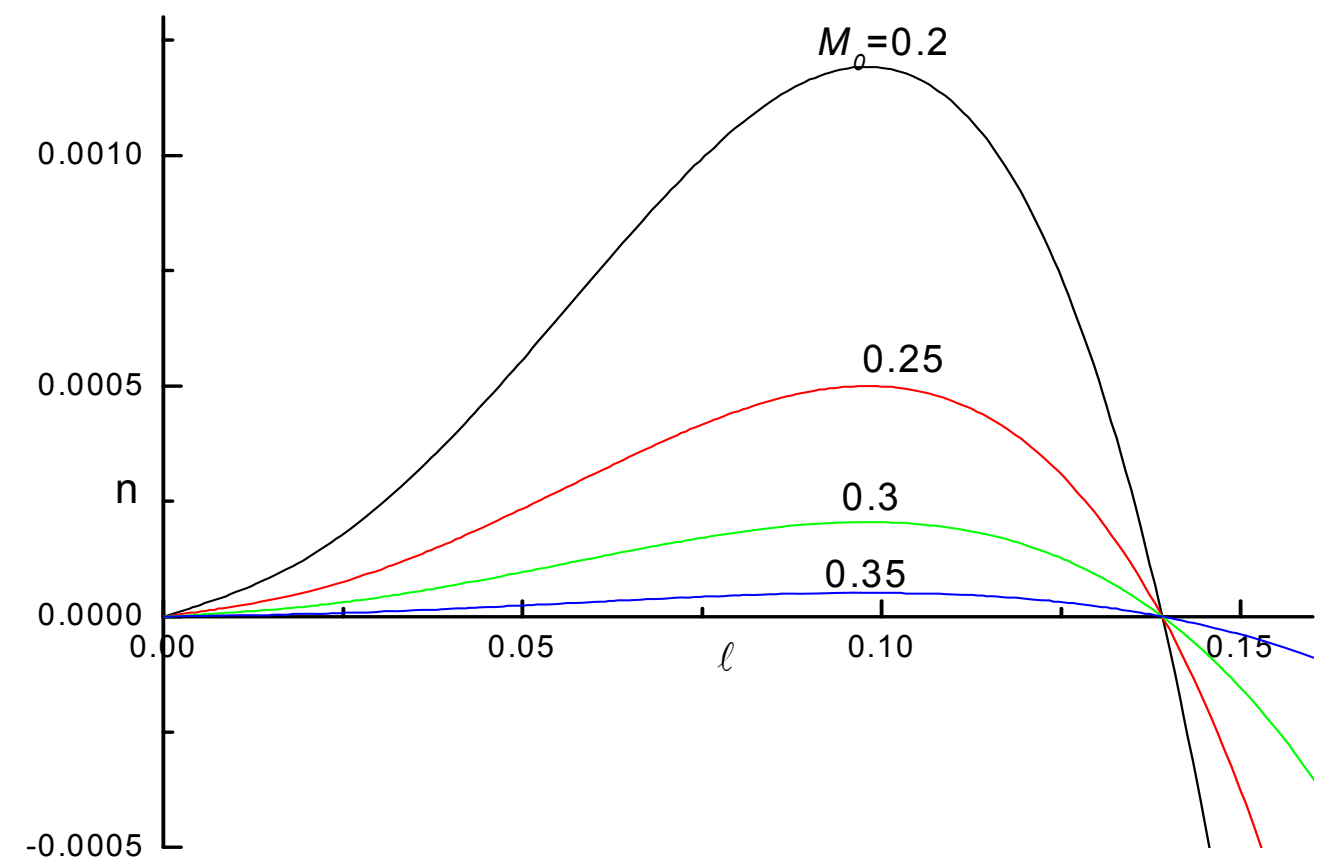

Fig.2. Growth rate, $n$ versus the wave number, $\ell$ for different values of the Hartmann number, $M$ when $\alpha_{p}=0.1, \sigma_{p}=4.0, \mathrm{M}=5$ and $B=0.02$. 
However, in order to understand the effect of the magnetic field on instability, we now fix values of other parameters $\alpha_{p}=0.1, \sigma_{p}=4, B=0.02$ and $\mathrm{M}=0.3$ and vary the ratios of the Hartmann number $\mathrm{M}$ as shown in Fig.3. In other words, the RT instability can be suppressed by the magnetic field. Increasing the Hartmann ratio results in slightly increasing the critical wave number and decreasing the maximum growth rate. We observe that the magnetic field has a stabilization effect on the RT instability for the selected values of input parameters due to the increased Hartmann ratio (Lorentz force to viscous force).

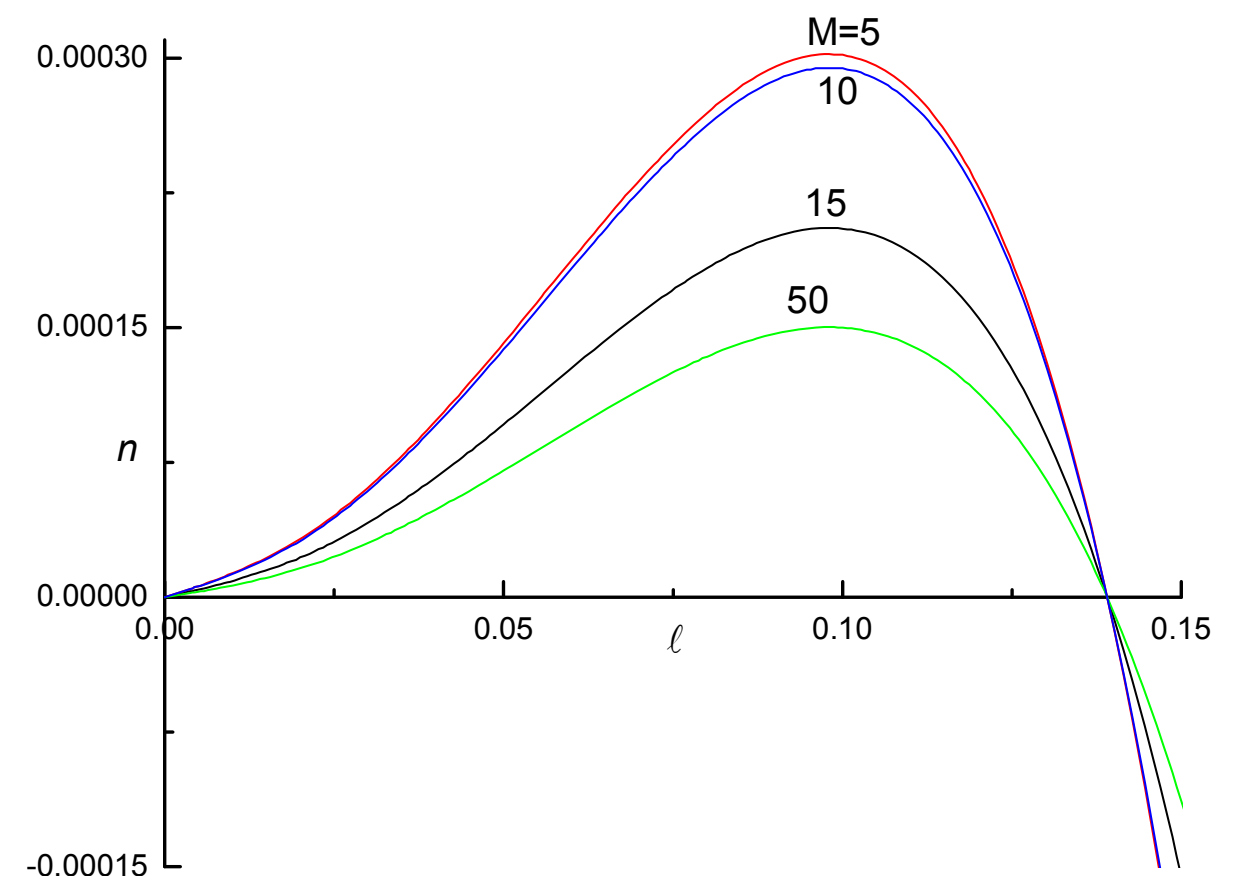

Fig.3. Growth rate, $n$ versus the wave number, $\ell$ for different values of the Hartmann number, $M$ when $\alpha_{p}=0.1, \sigma_{p}=4.0, M_{0}=0.3$ and $B=0.02$.

In addition, we have investigated the effect of the surface tension of the fluid on the instability of the interface. In our sample calculations, we have taken $\alpha_{p}=0.1, \sigma_{p}=4, M_{0}=0.3, \mathrm{M}=5$ and varied the Bond number $B$. For this input parameters, the critical wave number and maximum growth rate decreased as the ratio of the Bond number B decreased from 0.4 to 0.1 as observed in Fig.4.

Finally, we can understand the effect of the porous properties on instability for fixed values of other parameters $\alpha_{p}=0.1, B=0.02, M_{0}=0.3, \mathrm{M}=5$ and varying the ratios of the porous parameters.

Figure 4 displays the results of our calculations, showing that increasing the ratio of porous parameters $\sigma_{p}$ from 4 to 50 and thus increasing the Darcy resistance compared to the viscous force) increase the critical wavelength and decrease the maximum growth rate, thus having a stabilizing effect. We conclude that an increase in $\sigma_{p}$ also stabilizes the RTI due to the resistance offered by the solid particles of the porous layer to the fluid. 


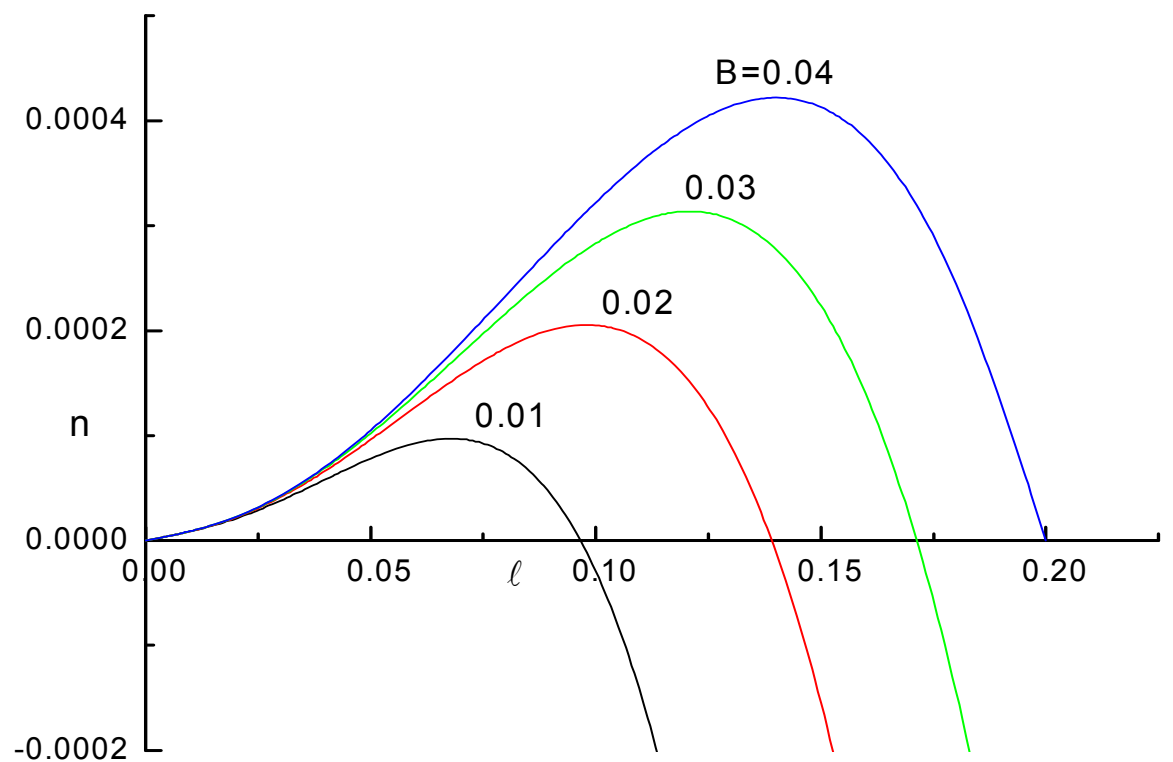

Fig.4. Growth rate, $n$ versus the wave number, $\ell$ for different values of the Bond number, $\mathrm{B}$ when $\alpha_{p}=0.1, \sigma_{p}=4.0, M_{0}=0.3$ and $\mathrm{M}=5$.

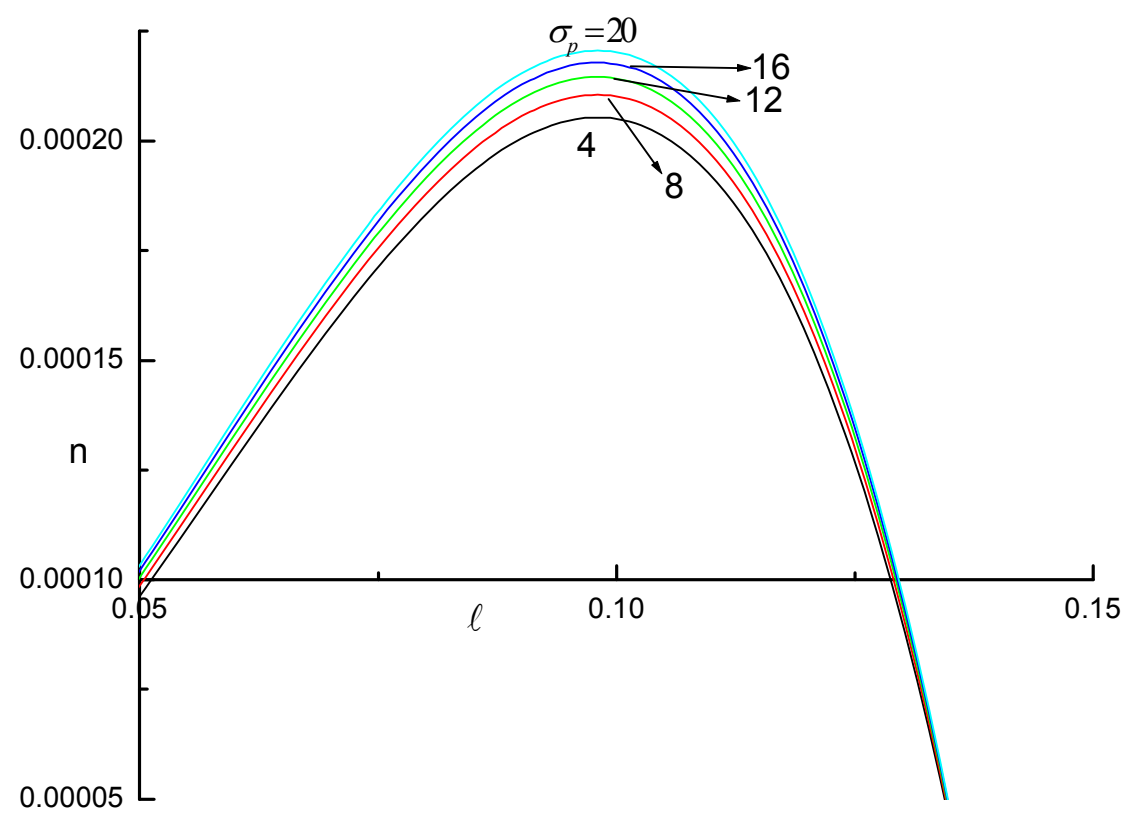

Fig.5. Growth rate, $n$ versus the wave number, $\ell$ for different values of the porous parameter $\sigma_{p}$ when $\alpha_{p}=0.1, B=0.02, M_{0}=0.3$ and $\mathrm{M}=5$. 


\section{Conclusion}

An analytical growth rate is derived for the RT instability by taking into account the magnetic field and surface tension in a couple-stress fluid. It is found that the magnetic field and porous parameter (porosity) have a stabilization effect whereas the buoyancy force has a destabilization effect on the RT instability in the presence of a sharp interface.

1. The RT instability only occurs in the long wave region and can be completely suppressed if the stabilizing effect of the magnetic field dominates.

2. The RT instability increases with the wave number and flow shear, and acts much like a KelvinHelmholtz instability when a destabilizing effect of sheared flow dominates.

If the wave number and the flow shear are relatively small, the growth rate of the RT instability rises monotonically. Otherwise, the growth rate firstly increases, achieves its maximum, and then falls down as density difference increases.

We have studied the linear stability of a two-fluid flow in a channel where the fluids are assumed to be Newtonian with different fluid properties (Hartmann number, couple-stress parameter, surface tension and porous parameter) and subjected to a magnetic field normal to their interface. For this purpose, we have derived and then linearized the equations of motion where the interaction between the hydrodynamic and electric problems occurs through the stress balance at the fluid interface. The growth rate of the perturbation was then computed by using the normal mode method and its variation studied as a function of the dimensionless parameters i.e., the Hartmann M, couple-stress parameter $M_{0}$ as well as the Bond number B and porous parameter $\sigma_{p}$. While two layer flows in channels of small dimensions are rather stable, instability of the fluid-porous interface is highly desirable in certain cases, particularly for chemical industry, in petroleum production engineering applications where the mixing of reagents are crucial steps in the process. However, in systems of larger scale, instability of the fluid-porous interface in a channel is often an undesired physical phenomenon. In such situations, controlling the flow requires stabilization of the interface. In searching for a method capable of either stabilizing a potentially unstable interface or destabilizing a potentially stable one, we have investigated the role of the magnetic field on the two-layer channel flow problem and demonstrated that either destabilization or stabilization can be obtained and presented by growth rates in situations where the magnetic field is stabilizing or destabilizing over a broad range of wave numbers. For increasing the Hartmann number M. The degree of the fluid mixing between the two layers is strongly influenced by the magnetic field strength and the RT instability can be suppressed by the magnetic field.

The variation in the Bond number decreases the growth rate and hence makes the interface more stable. Also we conclude that the increase in the porous parameter is to decrease the growth rate showing thereby the stabilizing effect on the interface. It is found that the magnetic field and couple-stress have a stabilization effect whereas the buoyancy force (surface tension) has a destabilization effect on the RT instability in the presence of porous media.

\section{Acknowledgement}

This work is supported by the VGST, Department of Science \& Technology, Government of Karnataka under grant no. GRD-433/2014-15. The authors (VBA, MN, PMG) wish to thank the VC/DCE/Principal for the support and encouragement.

\section{Nomenclature}

$$
\begin{aligned}
& \text { B - Bond number }\left(\delta h^{2} / \gamma\right) \\
& \text { B - magnetic induction } \\
& \text { D - dielectric field }
\end{aligned}
$$




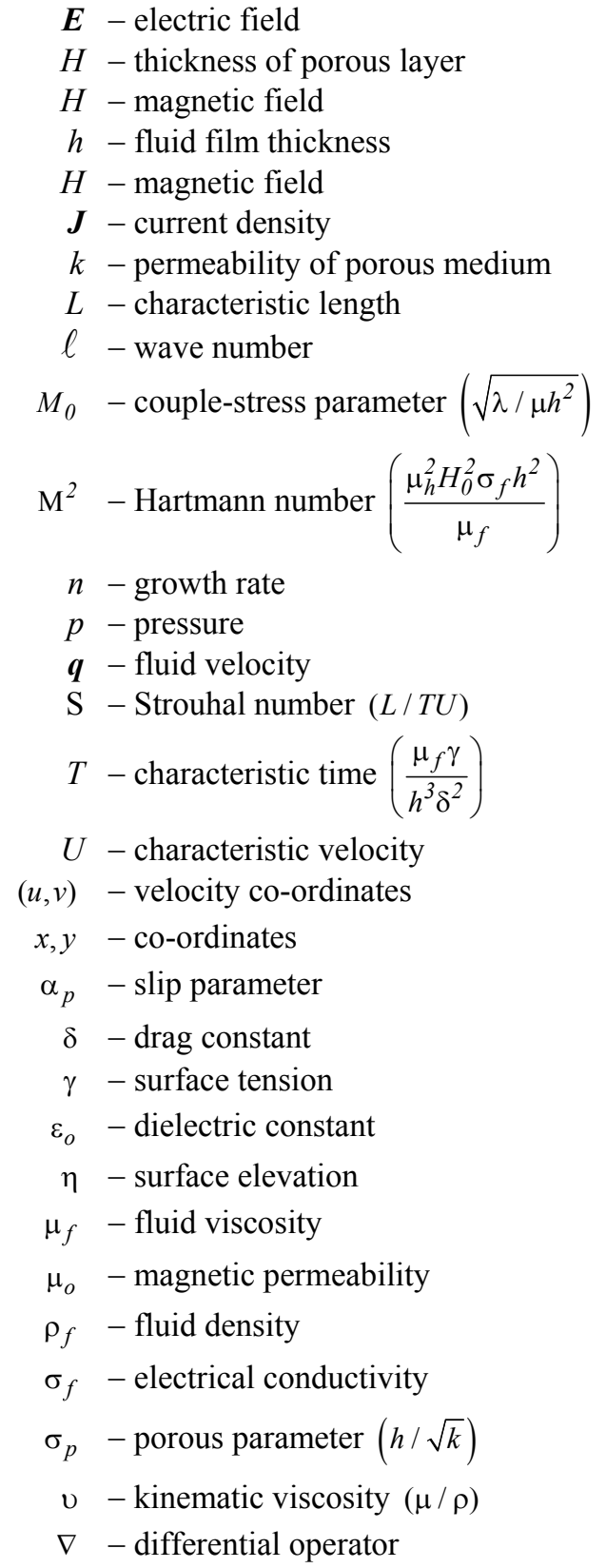

\section{Subscript}

$$
\begin{array}{ll}
f & - \text { fluid } \\
p & - \text { porous }
\end{array}
$$

\section{References}

[1] Shilling O., Steinkamp M. and Baer M. (2006): Turbulent Mixing and Hydrodnamics. - Predictive Science Academic Alliances Program Technical White Paper.

[2] Sharp D.H. (1984): An overview of Rayleigh-Taylor instability. - Physica D 12, pp.3-18. 
[3] Rayleigh L. (1883): On the stability or instability of certain fluid motion. - Proc. Lond. Math., Soc., vol.11, No.57.

[4] Taylor G.I. (1950): The instability of liquid surfaces when accelerated in a direction perpendicular to their planes I. - Proc. Roy. Soc. London, Ser. A201, pp.192-196.

[5] Chandrasekhar S. (1961): Hydrodynamic and Hydromagnetic Stability. - New York: Dover Publications.

[6] Bhatia P.K. (1974): Rayleigh-Taylor instability of two viscous superposed conducting fluids. - Nuovo Cim. 19B, pp.161-168.

[7] Sharma R.C. and Sharma K.C. (1978): Rayleigh-Taylor instability of two superposed conducting fluids in the presence of suspended particles. - Acta. Physica Hungarica 45, pp.213-220.

[8] Stokes V.K. (1966): Couple stresses in Fluids. - Phys. Fluids, vol.9, pp.1709-1715.

[9] Sinha P., Singh P.C. and Prasad K.R. (1981): Couple stresses in journal bearings lubricants and the effect of cavitation. - Wear, vol.67, No.1, pp.15-24.

[10] Bujurke N.M. and Jayaraman G. (1982): The influence of couple stresses in squeeze films. - Int. J. Mech. Sci., vol.24, pp.369-376.

[11] Walicki E. and Walicka A. (1999): Inertia effect in the squeeze film of a couple-stress fluid in biological bearings. - Appl. Mech. Engg., vol.4, No.2, pp.363-373.

[12] Sunil, Sharma R.C. and Chandel R.S. (2002): On superposed fluids in porous medium in hydrodynamics. - Z. Naturforsch, 57a, pp.955-960.

[13] Chavaraddi K.B., Awati V.B. and Gouder P.M. (2013): Effect Boundary Roughness on Rayleigh-Taylor Instability of a Couple-Stress Fluid. - Gen. Math. Notes, vol.17, No.2, pp.66-75.

[14] Srinivasan B., Dimonte G. and Tang X.-Z. (2012): Magnetic field generation in Rayleigh-Taylor unstable inertial confinement fusion plasmas. - Phys. Rev. Lett. 108, 165002.

[15] Srinivasan B. and Tang X.-Z. (2012): Mechanism for magnetic field generation and growth in Rayleigh-Taylor unstable inertial confinement fusion plasmas. - Phys. Plasmas 19, 082703.

[16] Modica F., Plewa T. and Zhiglo A. (2013): The Braginskii model of the Rayleigh--Taylor instability. I. Effects of self-generated magnetic fields and thermal conduction in two dimensions. - High Energy Density Phys., vol.9, pp.767-780.

[17] Alves E.P., Grismayer T., Martins S.F., Fiza F., Fonseca R.A. and Silva L.O. (2012): Large-scale magnetic field generation via the kinetic Kelvin-Helmholtz instability in unmagnetized scenarios. - Astrophys. J. Lett. 746:L14.

Received: March 31, 2017

Revised: March 20, 2018 